\title{
Determinization of fuzzy automata by means of the degrees of language inclusion
}

\author{
Ivana Micić, Zorana Jančić, Jelena Ignjatović, and Miroslav Ćirić
}

\begin{abstract}
Determinization of fuzzy finite automata is understood here as a procedure of their conversion into equivalent crisp-deterministic fuzzy automata, which can be viewed as being deterministic with possibly infinitely many states, but with fuzzy sets of terminal states. Particularly significant determinization methods are those that provide a minimal crisp-deterministic fuzzy automaton equivalent to the original fuzzy finite automaton, called canonization methods. One canonization method for fuzzy finite automata, the Brzozowski type determinization, has been developed recently by Jančić and Ćirić in [10]. Here we provide another canonization method for a fuzzy finite automaton $\mathcal{A}=(\mathcal{A}, \sigma, \delta, \tau)$ over a complete residuated lattice $\mathcal{L}$, based on the degrees of inclusion of the right fuzzy languages associated with states of $\mathcal{A}$ into the left derivatives of the fuzzy language recognized by $\mathcal{A}$. The proposed procedure terminates in a finite number of steps whenever the membership values taken by $\delta, \sigma$ and $\tau$ generate a finite subsemiring of the semiring reduct of $\mathcal{L}$. This procedure is generally faster than the Brzozowski type determinization, and if the basic operations in the residuated lattice $\mathcal{L}$ can be performed in constant time, it has the same computational time as all other determinization procedures provided in [8], [11], [12].
\end{abstract}

\section{Index Terms}

Fuzzy automaton; Complete residuated lattice; Crisp-deterministic automaton; Determinization; Inclusion degree;

\section{InTRODUCTION}

Many practical applications of automata require determinization, a procedure of converting a nondeterministic finite automaton to an equivalent deterministic finite automaton. In the context of fuzzy automata, determinization is generally understood as conversion of a fuzzy finite automaton to an equivalent crisp-deterministic fuzzy automaton, which can be viewed as being deterministic with possibly infinitely many states, but with a fuzzy set of terminal states. The standard determinization method, known as the subset construction, converts a nondeterministic automaton with $n$ states to an equivalent deterministic automaton with up to $2^{n}$ states, and the fuzzy version of this construction may even give an infinite crispdeterministic fuzzy automaton. That is why an extremely important task is to find such methods that will mitigate the potential enormous growth of the number of states during the determinization. Particularly significant are those determinization methods that provide minimal crisp-deterministic fuzzy automata, called canonization methods. One canonization method for fuzzy finite automata, the Brzozowski type determinization, has been developed recently by Jančić and Ćirić in [10]. The purpose of this paper is to provide another canonization method for fuzzy finite automata over a complete residuated lattice.

Determinization of fuzzy automata was first studied by Bělohlávek [2], in the context of fuzzy finite automata over a complete distributive lattice, an Li and Pedrycz [13], in the context of fuzzy finite automata over a lattice-ordered monoid. Determinization algorithms that were provided there generalize

Research supported by Ministry Education and Science, Republic of Serbia, Grant No. 174013

I. Micić, Z. Jančić, J. Ignjatović, and M. Ćirić are with the University of Niš, Faculty of Sciences and Mathematics, Department of Computer Science, Višegradska 33, 18000 Niš, Serbia

E-mail: ivanajancic84@gmail.com, zoranajancic329@gmail.com, jelena.ignjatovic@pmf.edu.rs, miroslav.ciric@pmf.edu.rs

Manuscript received April, 2014 
the subset construction. Another algorithm, provided by Ignjatović et al. [8], also generalizes the subset construction and for any input produces a smaller crisp-deterministic fuzzy automaton than algorithms from [2], [13]. This crisp-deterministic fuzzy automaton can be alternatively constructed by means of the Nerode right congruence of the original fuzzy finite automaton, and it was called in [9] the Nerode automaton of the original fuzzy finite automaton. The Nerode automaton was constructed in [8] for fuzzy finite automata over a complete residuated lattice, and it was noted that the identical construction can also be made in a more general context, for fuzzy finite automata over a lattice-ordered monoid, and even for weighted finite automata over a semiring. The same construction was also transferred in [4] to weighted automata over strong bimonoids. The algorithm proposed by Jančić et al. in [11], which generalizes the "transition sets construction" given in [17], [18], produces a crisp-deterministic fuzzy or weighted automaton that is even smaller than the Nerode automaton, and further progress has been made in a recent paper by Jančić et al. [12], where algorithms which perform both determinization and state reduction have been provided. In addition, Jančić and Ćirić in [10] adapted the well-known Brzozowski's double reversal determinization algorithm to fuzzy automata and provided a Brzozowski type determinization algorithm that yields a minimal crisp-deterministic fuzzy automaton equivalent to the original fuzzy finite automaton. It is important to note that another form of determinization, more general than crisp-determinization, has recently been studied by González de Mendivil and Garitagoitia [5], [6].

In the present paper we propose another determinization method which for any fuzzy finite automaton $\mathcal{A}=(A, \sigma, \delta, \tau)$ over a complete residuated lattice $\mathcal{L}$ produces a minimal crisp-deterministic fuzzy automaton $\mathcal{A}_{d}$ equivalent to $\mathcal{A}$. The automaton $\mathcal{A}_{d}$ does not necessarily have a finite number of states, but whenever the membership values taken by $\delta, \sigma$ and $\tau$ generate a finite subsemiring of the semiring reduct of $\mathcal{L}$, then the number of states of $\mathcal{A}_{d}$ is also finite. The proposed canonization procedure is based on the degrees of inclusion of the right fuzzy languages associated with states of $\mathcal{A}$ into the left derivatives of the fuzzy language recognized by $\mathcal{A}$. The computation time of this procedure is generally better than the computation time of the Brzozowski type determinization, and if the basic operations in the underlying residuated lattice can be performed in constant time, it has the same computational time as all other determinization procedures provided in [8], [11], [12].

The paper is organized as follows. In Section 2 we recall basic notions and notation concerning fuzzy sets and relations, fuzzy automata and languages, crisp-deterministic fuzzy automata, and the Nerode and reverse Nerode automaton. Our main theoretical results are presented in Section 3, where for a given fuzzy finite automaton we construct an equivalent accessible crisp-deterministic fuzzy automaton whose states are fuzzy sets defined by means of the degrees of inclusion of certain fuzzy languages. In Section 4 we state an algorithm which constructs this crisp-deterministic fuzzy automaton, perform the analysis of the computation time, and give examples that demonstrates the application of the algorithm.

\section{Preliminaries}

\subsection{Fuzzy sets and relations}

In this paper we use complete residuated lattices as structures of membership values. A residuated lattice is an algebra $\mathcal{L}=(L, \wedge, \vee, \otimes, \rightarrow, 0,1)$ such that

(L1) $(L, \wedge, \vee, 0,1)$ is a lattice with the least element 0 and the greatest element 1 ,

(L2) $(L, \otimes, 1)$ is a commutative monoid with the unit 1 ,

$(\mathrm{L} 3) \otimes$ and $\rightarrow$ form an adjoint pair, i.e., they satisfy the adjunction property: for all $x, y, z \in L$,

$$
x \otimes y \leqslant z \Leftrightarrow x \leqslant y \rightarrow z .
$$

If, additionally, $(L, \wedge, \vee, 0,1)$ is a complete lattice, then $\mathcal{L}$ is called a complete residuated lattice.

The algebra $\mathcal{L}^{*}=(L, \vee, \otimes, 0,1)$ is a semiring, and it is called the semiring reduct of $\mathcal{L}$.

The operations $\otimes$ (called multiplication) and $\rightarrow$ (called residuum) are intended for modeling the conjunction and implication of the corresponding logical calculus, and supremum $(\bigvee)$ and infimum $(\wedge)$ are intended for modeling of the existential and general quantifier, respectively. An operation $\leftrightarrow$ defined by

$$
x \leftrightarrow y=(x \rightarrow y) \wedge(y \rightarrow x),
$$


called biresiduum (or biimplication), is used for modeling the equivalence of truth values. It can be easily shown that with respect to $\leqslant, \otimes$ is isotonic in both arguments, $\rightarrow$ is isotonic in the second and antitonic in the first argument, and for any $x, y, z \in L$ the following hold:

$$
\begin{aligned}
& (x \rightarrow y) \otimes x \leqslant y, \\
& y \leqslant x \rightarrow(x \otimes y),
\end{aligned}
$$

For other properties of complete residuated lattices one can refer to [1], [3].

The most studied and applied structures of truth values, defined on the real unit interval $[0,1]$ with $x \wedge y=\min (x, y)$ and $x \vee y=\max (x, y)$, are the Eukasiewicz structure $(x \otimes y=\max (x+y-1,0), x \rightarrow y=$ $\min (1-x+y, 1))$, the Goguen (product) structure $(x \otimes y=x \cdot y, x \rightarrow y=1$ if $x \leqslant y$ and $=y / x$ otherwise) and the Gödel structure $(x \otimes y=\min (x, y), x \rightarrow y=1$ if $x \leqslant y$ and $=y$ otherwise). Another important set of truth values is the set $\left\{a_{0}, a_{1}, \ldots, a_{n}\right\}, 0=a_{0}<\cdots<a_{n}=1$, with $a_{k} \otimes a_{l}=a_{\max (k+l-n, 0)}$ and $a_{k} \rightarrow a_{l}=a_{\min (n-k+l, n)}$. A special case of the latter algebras is the two-element Boolean algebra of classical logic with the support $\{0,1\}$. The only adjoint pair on the two-element Boolean algebra consists of the classical conjunction and implication operations. This structure of truth values is called the Boolean structure.

In the sequel $\mathcal{L}$ will be a complete residuated lattice. A fuzzy subset of a set $A$ over $\mathcal{L}$, or simply a fuzzy subset of $A$, is any mapping from $A$ into $L$. Ordinary crisp subsets of $A$ are considered as fuzzy subsets of $A$ taking membership values in the set $\{0,1\} \subseteq L$. Let $f$ and $g$ be two fuzzy subsets of $A$. The equality of $f$ and $g$ is defined as the usual equality of mappings, i.e., $f=g$ if and only if $f(x)=g(x)$, for every $x \in A$. The inclusion $f \leqslant g$ is also defined pointwise: $f \leqslant g$ if and only if $f(x) \leqslant g(x)$, for every $x \in A$. Endowed with this partial order the set $L^{A}$ of all fuzzy subsets of $A$ forms a complete residuated lattice, in which the meet (intersection) $\bigwedge_{i \in I} f_{i}$ and the join (union) $\bigvee_{i \in I} f_{i}$ of an arbitrary family $\left\{f_{i}\right\}_{i \in I}$ of fuzzy subsets of $A$ are mappings from $A$ into $L$ defined by

$$
\left(\bigwedge_{i \in I} f_{i}\right)(x)=\bigwedge_{i \in I} f_{i}(x), \quad\left(\bigvee_{i \in I} f_{i}\right)(x)=\bigvee_{i \in I} f_{i}(x),
$$

and the product $f \otimes g$ is a fuzzy subset defined by $f \otimes g(x)=f(x) \otimes g(x)$, for each $x \in A$. The degree of inclusion $I(f, g)$ of two fuzzy sets $f, g \in L^{A}$, in that order (or the degree of inclusion of $f$ into $g$ ), is defined by

$$
I(f, g)=\bigwedge_{a \in A} f(a) \rightarrow g(a) .
$$

In other words, $I(f, g)$ is a measure of "how much $f$ is contained in $g$ ".

A fuzzy relation between sets $A$ and $B$ (in this order) is any mapping from $A \times B$ to $L$, i.e. , any fuzzy subset of $A \times B$, and the equality, inclusion (ordering), joins and meets of fuzzy relations are defined as for fuzzy sets. Set of all fuzzy relations between $A$ and $B$ will be denoted by $L^{A \times B}$. In particular, a fuzzy relation on a set $A$ is any function from $A \times A$ to $L$, i.e., any fuzzy subset of $A \times A$. The set of all fuzzy relations on $A$ will be denoted by $L^{A \times A}$. The reverse or inverse of a fuzzy relation $\alpha \in L^{A \times B}$ is a fuzzy relation $\alpha^{-1} \in L^{B \times A}$ defined by $\alpha^{-1}(b, a)=\alpha(a, b)$, for all $a \in A$ and $b \in B$. A crisp relation is a fuzzy relation which takes values only in the set $\{0,1\}$, and if $\alpha$ is a crisp relation of $A$ to $B$, then expressions " $\alpha(a, b)=1 "$ and " $(a, b) \in \alpha$ " will have the same meaning.

For non-empty sets $A, B$ and $C$, and fuzzy relations $\alpha \in L^{A \times B}$ and $\beta \in L^{B \times C}$, their composition $\alpha \circ \beta \in L^{A \times C}$ is a fuzzy relation defined by

$$
(\alpha \circ \beta)(a, c)=\bigvee_{b \in B} \alpha(a, b) \otimes \beta(b, c),
$$

for all $a \in A$ and $c \in C$. For $f \in L^{A}, \alpha \in L^{A \times B}$ and $g \in L^{B}$, compositions $f \circ \alpha \in L^{B}$ and $\alpha \circ g \in L^{A}$ are fuzzy sets defined by

$$
(f \circ \alpha)(b)=\bigvee_{a \in A} f(a) \otimes \alpha(a, b), \quad(\alpha \circ g)(a)=\bigvee_{b \in B} \alpha(a, b) \otimes g(b)
$$


for every $a \in A$ and $b \in B$. Finally, the composition of two fuzzy sets $f, g \in L^{A}$ is an element $f \circ g \in L$ (scalar) defined by

$$
f \circ g=\bigvee_{a \in A} f(a) \otimes g(a)
$$

When the underlying sets are finite, fuzzy relations can be interpreted as matrices and fuzzy sets as vectors with entries in $L$, and then the composition of fuzzy relations can be interpreted as the matrix product, compositions of fuzzy sets and fuzzy relations as vector-matrix products, and the composition of two fuzzy set as the scalar (dot) product.

It is easy to verify that the composition of fuzzy relations is associative, i.e.,

$$
(\alpha \circ \beta) \circ \gamma=\alpha \circ(\beta \circ \gamma),
$$

for all $\alpha \in L^{A \times B}, \beta \in L^{B \times C}$ and $\gamma \in L^{C \times D}$, and

$$
(f \circ \alpha) \circ \beta=f \circ(\alpha \circ \beta), \quad(f \circ \alpha) \circ g=f \circ(\alpha \circ g), \quad(\alpha \circ \beta) \circ h=\alpha \circ(\beta \circ h)
$$

for all $\alpha \in L^{A \times B}, \beta \in L^{B \times C}, f \in L^{A}, g \in L^{B}$ and $h \in L^{C}$. Hence, all parentheses in (8) and (9) can be omitted.

\subsection{Fuzzy automata}

Throughout this paper, $\mathbb{N}$ denotes the set of natural numbers (without zero), $X$ is an (finite) alphabet, $X^{+}$ and $X^{*}$ denote, respectively, the free semigroup and the free monoid over $X, \varepsilon$ denotes the empty word in $X^{*}$, and if not noted otherwise, $\mathcal{L}$ is a complete residuated lattice.

A fuzzy automaton over $\mathcal{L}$ and $X$, or simply a fuzzy automaton, is a quadruple $\mathcal{A}=(A, \sigma, \delta, \tau)$, where $A$ is a non-empty set, called the set of states, $\delta: A \times X \times A \rightarrow L$ is a fuzzy subset of $A \times X \times A$, called the fuzzy transition function, and $\sigma: A \rightarrow L$ and $\tau: A \rightarrow L$ are fuzzy subsets of $A$, called the fuzzy set of initial states and the fuzzy set terminal states, respectively. We can interpret $\delta(a, x, b)$ as the degree to which an input letter $x \in X$ causes a transition from a state $a \in A$ into a state $b \in A$, and we can interpret $\sigma(a)$ and $\tau(a)$ as the degrees to which $a$ is respectively an input state and a terminal state. For methodological reasons we allow the set of states $A$ to be infinite. A fuzzy automaton whose set of states is finite is called a fuzzy finite automaton. A fuzzy automaton over the Boolean structure is called a nondeterministic automaton or a Boolean automaton.

Define a family $\left\{\delta_{x}\right\}_{x \in X}$ of fuzzy relations on $A$ by $\delta_{x}(a, b)=\delta(a, x, b)$, for each $x \in X$, and all $a, b \in A$, and extend this family to the family $\left\{\delta_{u}\right\}_{u \in X^{*}}$ inductively, as follows: $\delta_{\varepsilon}=\Delta_{A}$, where $\Delta_{A}$ is the crisp equality relation on $A$, and

$$
\delta_{x_{1} x_{2} \ldots x_{n}}=\delta_{x_{1}} \circ \delta_{x_{2}} \circ \cdots \circ \delta_{x_{n}}
$$

for all $n \in \mathbb{N}, x_{1}, x_{2}, \ldots, x_{n} \in X$. Members of this family are called fuzzy transiton relations of $\mathcal{A}$. Evidently, $\delta_{u v}=\delta_{u} \circ \delta_{v}$, for all $u, v \in X^{*}$. In addition, define families $\left\{\sigma_{u}\right\}_{u \in X^{*}}$ and $\left\{\tau_{u}\right\}_{u \in X^{*}}$ by

$$
\sigma_{u}=\sigma \circ \delta_{u}, \quad \tau_{u}=\delta_{u} \circ \tau
$$

for all $u \in X^{*}$. In dealing with fuzzy finite automata, fuzzy transition relations $\left\{\delta_{u}\right\}_{u \in X^{*}}$ are represented by fuzzy matrices with entries in $L$, whereas fuzzy sets $\left\{\sigma_{u}\right\}_{u \in X^{*}}$ are represented by row vectors and $\left\{\tau_{u}\right\}_{u \in X^{*}}$ by column vectors with entries in $L$.

A fuzzy language in $X^{*}$ over $\mathcal{L}$, or just a fuzzy language, is any fuzzy subset of $X^{*}$, i.e., any function from $X^{*}$ into L. A fuzzy language recognized by a fuzzy automaton $\mathcal{A}=(A, \sigma, \delta, \tau)$ is a fuzzy language $\llbracket \mathcal{A} \rrbracket \in L^{X^{*}}$ defined by

$$
\llbracket \mathcal{A} \rrbracket(u)=\bigvee_{a, b \in A} \sigma(a) \otimes \delta_{u}(a, b) \otimes \tau(b)=\sigma \circ \delta_{u} \circ \tau,
$$

for any $u \in X^{*}$. In other words, the membership degree of the word $u$ to the fuzzy language $\llbracket \mathcal{A} \rrbracket$ is equal to the degree to which $\mathcal{A}$ recognizes or accepts the word $u$. Fuzzy automata $\mathcal{A}$ and $\mathcal{B}$ are called language equivalent, or just equivalent, if $\llbracket \mathcal{A} \rrbracket=\llbracket \mathcal{B} \rrbracket$. 
The right fuzzy language associated with a state $a \in A$ is a fuzzy language $\tau_{a} \in L^{X^{*}}$ defined by

$$
\tau_{a}(u)=\tau_{u}(a)=\bigvee_{b \in A} \delta_{u}(a, b) \otimes \tau(b),
$$

for all $u \in X^{*}$, i.e., $\tau_{a}$ is the fuzzy language recognized by a fuzzy automaton $\mathcal{A}_{a}=(A, \delta, a, \tau)$ obtained from $\mathcal{A}$ by replacing $\sigma$ with the single crisp initial state $a$.

Cardinality of a fuzzy automaton $\mathcal{A}=(A, \sigma, \delta, \tau)$, in notation $|\mathcal{A}|$, is defined as the cardinality of its set of states $A$. A fuzzy automaton $\mathcal{A}$ is called minimal fuzzy automaton of a fuzzy language $f \in L^{X^{*}}$ if $\llbracket \mathcal{A} \rrbracket=f$ and $|\mathcal{A}| \leqslant\left|\mathcal{A}^{\prime}\right|$, for every fuzzy automaton $\mathcal{A}^{\prime}$ such that $\llbracket \mathcal{A}^{\prime} \rrbracket=f$. A minimal fuzzy automaton recognizing a given fuzzy language $f$ is not necessarily unique up to an isomorphism. This is also true for nondeterministic automata.

Let $\mathcal{A}=(A, \delta, \sigma, \tau)$ be a fuzzy automaton over $\mathcal{L}$ and $X$. The reverse fuzzy automaton of $\mathcal{A}$ is a fuzzy automaton $\overline{\mathcal{A}}=(A, \bar{\delta}, \bar{\sigma}, \bar{\tau})$, where $\bar{\sigma}=\tau, \bar{\tau}=\sigma$, and $\bar{\delta}: A \times X \times A \rightarrow L$ is defined by $\bar{\delta}^{A}(a, x, b)=\delta^{A}(b, x, a)$, for all $a, b \in A$ and $x \in X$. Roughly speaking, the reverse fuzzy automaton $\overline{\mathcal{A}}$ is obtained from $\mathcal{A}$ by exchanging fuzzy sets of initial and final states and "reversing" all the transitions. Due to the fact that the multiplication $\otimes$ is commutative, we have that $\bar{\delta}_{u}(a, b)=\delta_{\bar{u}}(b, a)$, for all $a, b \in A$ and $u \in X^{*}$.

The reverse fuzzy language of a fuzzy language $f \in L^{X^{*}}$ is a fuzzy language $\bar{f} \in L^{X^{*}}$ defined by $\bar{f}(u)=f(\bar{u})$, for each $u \in X^{*}$. As $\overline{(\bar{u})}=u$ for all $u \in X^{*}$, we have that $\overline{(\bar{f})}=f$, for any fuzzy language $f$. It is easy to see that the reverse fuzzy automaton $\overline{\mathcal{A}}$ recognizes the reverse fuzzy language $\overline{\llbracket \mathcal{A} \rrbracket}$ of the fuzzy language $\llbracket \mathcal{A} \rrbracket$ recognized by $\mathcal{A}$, i.e., $\llbracket \overline{\mathcal{A}} \rrbracket=\overline{\llbracket \mathcal{A} \rrbracket}$.

We can visualize a fuzzy finite automaton $\mathcal{A}=(A, \sigma, \delta, \tau)$ representing it as a labelled directed graph whose nodes are states of $\mathcal{A}$, an edge from a node $a$ to a node $b$ is labelled by pairs of the form $x / \delta_{x}(a, b)$, for any $x \in X$, and for any node $a$ we draw an arrow labelled by $\sigma(a)$ that enters this node, and an arrow labelled by $\tau(a)$ coming out of this node. For the sake of simplicity, we do not draw edges whose all labels are of the form $x / 0$, and incoming and outgoing arrows labelled by 0 . In particular, if $\mathcal{A}$ is a Boolean automaton, instead of any label of the form $x / 1$ we write just $x$, initial states are marked by incoming arrows without any label, and terminal states are marked by double circles.

For more information on fuzzy automata over complete residuated lattices we refer to [7], [8], [9], [14], [15], [16], [19], [20].

\subsection{Crisp-deterministic fuzzy automata}

Let $\mathcal{A}=(A, \sigma, \delta, \tau)$ be a fuzzy automaton over $X$ and $\mathcal{L}$. The fuzzy transition function $\delta$ is called crispdeterministic if for every $x \in X$ and every $a \in A$ there exists $a^{\prime} \in A$ such that $\delta_{x}\left(a, a^{\prime}\right)=1$, and $\delta_{x}(a, b)=0$, for all $b \in A \backslash\left\{a^{\prime}\right\}$. The fuzzy set of initial states $\sigma$ is called crisp-deterministic if there exists $a_{0} \in A$ such that $\sigma\left(a_{0}\right)=1$, and $\sigma(a)=0$, for every $a \in A \backslash\left\{a_{0}\right\}$. If both $\sigma$ and $\delta$ are crisp-deterministic, then $\mathcal{A}$ is called a crisp-deterministic fuzzy automaton (for short: $c d f a$ ), and if it is finite, then it is called a crisp-deterministic fuzzy finite automaton (for short: $c d f f a$ ).

A crisp-deterministic fuzzy automaton can also be defined as a quadruple $\mathcal{A}=\left(A, \delta, a_{0}, \tau\right)$, where $A$ is a non-empty set of states, $\delta: A \times X \rightarrow A$ is a transition function, $a_{0} \in A$ is an initial state and $\tau \in L^{A}$ is a fuzzy set of terminal states. The transition function $\delta$ can be extended to a function $\delta^{*}: A \times X^{*} \rightarrow A$ in the following way: $\delta^{*}(a, \varepsilon)=a$, for every $a \in A$, and $\delta^{*}(a, u x)=\delta\left(\delta^{*}(a, u), x\right)$, for all $a \in A, u \in X^{*}$ and $x \in X$. In this case the fuzzy language $\llbracket \mathcal{A} \rrbracket$ recognized by $\mathcal{A}$ is given by

$$
\llbracket \mathcal{A} \rrbracket(u)=\tau\left(\delta^{*}\left(a_{0}, u\right)\right),
$$

for every $u \in X^{*}$. Clearly, the image of $\llbracket \mathcal{A} \rrbracket$ is contained in the image of $\tau$ which is finite if the set of

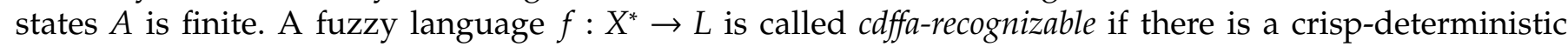
fuzzy finite automaton $\mathcal{A}$ over $X$ and $\mathcal{L}$ such that $\llbracket \mathcal{A} \rrbracket=f$. Then we say that $\mathcal{A}$ recognizes $f$.

A state $a \in A$ is called accessible if there exists $u \in X^{*}$ such that $\delta^{*}\left(a_{0}, u\right)=a$. If every state of $\mathcal{A}$ is accessible, then $\mathcal{A}$ is called an accessible crisp-deterministic fuzzy automaton. 
The initial state and transitions of a crisp-deterministic fuzzy automaton are graphically represented as in the case of Boolean automata, and the fuzzy set of terminal states is represented as in the case of fuzzy finite automata.

For a fuzzy language $f \in L^{X^{*}}$ and $u \in X^{*}$, we define a fuzzy language $f_{u} \in L^{X^{*}}$ by $f_{u}(v)=f(u v)$, for every $v \in X^{*}$. The fuzzy language $f_{u}$ is commonly called the left derivative of $f$ with respect to $u$, but for the sake of simplicity, $f_{u}$ will be called simply the derivative of $f$ with respect to $u$. Let $A_{f}=\left\{f_{u} \mid u \in X^{*}\right\}$ denote the set of all derivatives of $f$, and let $\delta_{f}: A_{f} \times X \rightarrow A_{f}$ and $\tau_{f}: A_{f} \rightarrow L$ be functions defined by

$$
\delta_{f}(g, x)=g_{x} \quad \text { and } \quad \tau_{f}(g)=g(\varepsilon),
$$

for all $g \in A_{f}$ and $x \in X$. Then $\mathcal{A}_{f}=\left(A_{f}, \delta_{f}, f, \tau_{f}\right)$ is an accessible crisp-deterministic fuzzy automaton, and it is called the derivative automaton of the fuzzy language $f$ [7], [9]. It was proved in [9] that the derivative automaton $\mathcal{A}_{f}$ is a minimal crisp-deterministic fuzzy automaton which recognizes $f$, and therefore, $\mathcal{A}_{f}$ is finite if and only if the fuzzy language $f$ is cdffa-recognizable. An algorithm for construction of the derivative automaton of a fuzzy language, based on simultaneous construction of the derivative automata of ordinary languages $f^{-1}(a)$, for all $a \in \operatorname{Im}(f)$, was also given in [9].

A crisp-deterministic fuzzy automaton $\mathcal{A}$ is called a minimal crisp-deterministic fuzzy automaton of a fuzzy language $f$ if $\llbracket \mathcal{A} \rrbracket=f$ and $|\mathcal{A}|<\left|\mathcal{A}^{\prime}\right|$, for any crisp-deterministic fuzzy automaton $\mathcal{A}^{\prime}$ such that $\llbracket \mathcal{A}^{\prime} \rrbracket=f$.

Further, the Nerode automaton of a fuzzy automaton $\mathcal{A}=(A, \sigma, \delta, \tau)$ is a crisp-deterministic fuzzy automaton $\mathcal{A}_{N}=\left(A_{N}, \sigma_{\varepsilon}, \delta_{N}, \tau_{N}\right)$, where $A_{N}=\left\{\sigma_{u} \mid u \in X^{*}\right\}$, and $\delta_{N}: A_{N} \times X \rightarrow A_{N}$ and $\tau_{N}: A_{N} \rightarrow L$ are defined, for all $u \in X^{*}$ and $x \in X$, by

$$
\delta_{N}\left(\sigma_{u}, x\right)=\sigma_{u x}, \quad \tau_{N}\left(\sigma_{u}\right)=\sigma_{u} \circ \tau,
$$

The Nerode automaton was first constructed in [8], where it was shown that the Nerode automaton of $\mathcal{A}$ is equivalent to $\mathcal{A}$, i.e., $\llbracket \mathcal{A}_{N} \rrbracket=\llbracket \mathcal{A} \rrbracket$. The name "Nerode automaton" was introduced in $[9]$.

The reverse Nerode automaton of a $\mathcal{A}$ is the Nerode automaton of the reverse automaton of $\mathcal{A}$, i.e., a crisp-deterministic fuzzy automaton $\mathcal{A}_{\bar{N}}=\left(A_{\bar{N}}, \tau_{\varepsilon}, \delta_{\bar{N}}, \tau_{\bar{N}}\right)$, where $A_{\bar{N}}=\left\{\tau_{u} \mid u \in X^{*}\right\}$, and $\delta_{\bar{N}}: A_{\bar{N}} \times X \rightarrow A_{\bar{N}}$ and $\tau_{\bar{N}}: A_{\bar{N}} \times L$ are defined by

$$
\delta_{\bar{N}}\left(\tau_{u}, x\right)=\tau_{x u}, \quad \tau_{\bar{N}}\left(\tau_{u}\right)=\sigma \circ \tau_{u},
$$

for all $u \in X^{*}$ and $x \in X$. As shown in [10], the reverse Nerode automaton of $\mathcal{A}$ is equivalent to the reverse fuzzy automaton of $\mathcal{A}$, i.e., $\llbracket \mathcal{A}_{\bar{N}} \rrbracket=\overline{\llbracket \mathcal{A} \rrbracket}$.

\section{The main Results}

Let $\mathcal{A}=(A, \delta, \sigma, \tau)$ be a fuzzy automaton over an alphabet $X$ and a complete residuated lattice $\mathcal{L}$. We define inductively a family $\left\{d_{u}\right\}_{u \in X^{*}}$ of fuzzy subsets of $A$ as follows: for the empty word $\varepsilon$ and $a \in A$ we set

$$
d_{\varepsilon}(a)=\bigwedge_{w \in X^{*}} \tau_{w}(a) \rightarrow \sigma \circ \tau_{w}
$$

and for all $u \in X^{*}, x \in X$ and $a \in A$ we set

$$
d_{u x}(a)=\bigwedge_{w \in X^{*}} \tau_{w}(a) \rightarrow d_{u} \circ \delta_{x} \circ \tau_{w}=\bigwedge_{w \in X^{*}} \tau_{w}(a) \rightarrow d_{u} \circ \tau_{x w} .
$$

The following theorem is one of the most important result of this paper.

Theorem 3.1: Let $\mathcal{A}=(A, \delta, \sigma, \tau)$ be a fuzzy automaton over an alphabet $X$ and a complete residuated lattice $\mathcal{L}$. Then for all $u, v \in X^{*}$ we have

$$
d_{u} \circ \tau_{v}=\sigma_{u} \circ \tau_{v}
$$


Proof: By induction on the length of the word $u$ we will prove that (17) is true for every $v \in X^{*}$. First, for the empty word $\varepsilon$ we have that

$$
d_{\varepsilon}(a)=\bigwedge_{w \in X^{*}} \tau_{w}(a) \rightarrow \sigma \circ \tau_{w} \leqslant \tau_{v}(a) \rightarrow \sigma \circ \tau_{v}
$$

holds for all $a \in A$ and $v \in X^{*}$, and according to (3),

$$
d_{\varepsilon} \circ \tau_{v}=\bigvee_{a \in A} d_{\varepsilon}(a) \otimes \tau_{v}(a) \leqslant \bigvee_{a \in A}\left(\tau_{v}(a) \rightarrow \sigma \circ \tau_{v}\right) \otimes \tau_{v}(a) \leqslant \sigma \circ \tau_{v}
$$

Therefore, $d_{\varepsilon} \circ \tau_{v} \leqslant \sigma \circ \tau_{v}$, for all $v \in X^{*}$.

Also, according to (4) and the fact that $\rightarrow$ is isotone in the second argument we have that

$$
\begin{aligned}
d_{\varepsilon}(a) & =\bigwedge_{w \in X^{*}} \tau_{w}(a) \rightarrow \sigma \circ \tau_{w}=\bigwedge_{w \in X^{*}}\left(\tau_{w}(a) \rightarrow\left(\bigvee_{b \in X^{*}} \sigma(b) \otimes \tau_{w}(b)\right)\right) \\
& \geqslant \bigwedge_{w \in X^{*}} \tau_{w}(a) \rightarrow\left(\sigma(a) \otimes \tau_{w}(a)\right) \geqslant \sigma(a),
\end{aligned}
$$

for all $a \in A$, so

$$
d_{\varepsilon} \circ \tau_{v}=\bigvee_{a \in A} d_{\varepsilon}(a) \otimes \tau_{v}(a) \geqslant \bigvee_{a \in A} \sigma(a) \otimes \tau_{v}(a)=\sigma \circ \tau_{v} .
$$

Therefore, $d_{\varepsilon} \circ \tau_{v}=\sigma \circ \tau_{v}$, for all $v \in X^{*}$, which means that (17) holds for $u=\varepsilon$ and every $v \in X^{*}$.

Let $u \in X^{*}$ be a word such that (17) holds for every word $v \in X^{*}$, and consider an arbitrary $x \in X$. Then

$$
d_{u x}(a)=\bigwedge_{w \in X^{*}} \tau_{w}(a) \rightarrow d_{u} \circ \delta_{x} \circ \tau_{w} \leqslant \tau_{v}(a) \rightarrow d_{u} \circ \delta_{x} \circ \tau_{v},
$$

for all $a \in A$ and $v \in X^{*}$, and according to (3),

$$
\begin{aligned}
d_{u x} \circ \tau_{v} & =\bigvee_{a \in A} d_{u x}(a) \otimes \tau_{v}(a) \leqslant \bigvee_{a \in A}\left(\tau_{v}(a) \rightarrow d_{u} \circ \delta_{x} \circ \tau_{v}\right) \otimes \tau_{v}(a) \\
& \leqslant d_{u} \circ \delta_{x} \circ \tau_{v}=d_{u} \circ \tau_{x v}=\sigma_{u} \circ \tau_{x v}=\sigma_{u x} \circ \tau_{v} .
\end{aligned}
$$

Next, according to (4) and the fact that $\rightarrow$ is isotone in the second argument we have that

$$
\begin{aligned}
d_{u x}(a) & =\bigwedge_{w \in X^{*}} \tau_{w}(a) \rightarrow d_{u} \circ \delta_{x} \circ \tau_{w}=\bigwedge_{w \in X^{*}}\left(\tau_{w}(a) \rightarrow\left(\bigvee_{b \in X^{*}}\left(d_{u} \circ \delta_{x}\right)(b) \otimes \tau_{w}(b)\right)\right) \\
& \geqslant \bigwedge_{w \in X^{*}} \tau_{w}(a) \rightarrow\left(\left(d_{u} \circ \delta_{x}\right)(a) \otimes \tau_{w}(a)\right) \geqslant\left(d_{u} \circ \delta_{x}\right)(a),
\end{aligned}
$$

for all $a \in A$, so

$$
\begin{aligned}
d_{u x} \circ \tau_{v} & =\bigvee_{a \in A} d_{u x}(a) \otimes \tau_{v}(a) \geqslant \bigvee_{a \in A}\left(d_{u} \circ \delta_{x}\right)(a) \otimes \tau_{v}(a) \\
& =d_{u} \circ \delta_{x} \circ \tau_{v}=d_{u} \circ \tau_{x v}=\sigma_{u} \circ \tau_{x v}=\sigma_{u x} \circ \tau_{v},
\end{aligned}
$$

and hence, $d_{u x} \circ \tau_{v}=\sigma_{u x} \circ \tau_{v}$. Thus, we conclude that (17) holds for all $u, v \in X^{*}$.

According to the previous theorem we have that

$$
d_{\varepsilon}(a)=\bigwedge_{w \in X^{*}} \tau_{w}(a) \rightarrow \sigma \circ \tau_{w}
$$

and

$$
d_{u x}(a)=\bigwedge_{w \in X^{*}} \tau_{w}(a) \rightarrow d_{u} \circ \tau_{x w}=\bigwedge_{w \in X^{*}} \tau_{w}(a) \rightarrow \sigma_{u} \circ \tau_{x w}=\bigwedge_{w \in X^{*}} \tau_{w}(a) \rightarrow \sigma_{u x} \circ \tau_{w}
$$

for all $a \in A, u \in X^{*}$ and $x \in X$, and hence

$$
d_{u}(a)=\bigwedge_{w \in X^{*}} \tau_{w}(a) \rightarrow \sigma_{u} \circ \tau_{w}=\bigwedge_{w \in X^{*}} \tau_{a}(w) \rightarrow f_{u}(w)=I\left(\tau_{a}, f_{u}\right)
$$


holds for all $u \in X^{*}$ and $a \in A$, where $f=\llbracket \mathcal{A} \rrbracket$. In other words, for all $u \in X^{*}$ and $a \in A$ we can understand $d_{u}(a)$ as the degree of inclusion of the right fuzzy language $\tau_{a}$ into the left derivative $f_{u}$ of the fuzzy language $f$ recognized by $\mathcal{A}$.

Now, set $A_{d}=\left\{d_{u} \mid u \in X^{*}\right\}$ and define functions $\delta_{d}: A_{d} \times X \rightarrow A_{d}$ and $\tau_{d}: A_{d} \rightarrow L$ by

$$
\delta_{d}\left(d_{u}, x\right)=d_{u x}, \quad \tau_{d}\left(d_{u}\right)=d_{u} \circ \tau,
$$

for all $d_{u} \in A_{d}$ and $x \in X$.

We have the following:

Theorem 3.2: Let $\mathcal{A}=(A, \delta, \sigma, \tau)$ be a fuzzy automaton over an alphabet $X$ and a complete residuated lattice $\mathcal{L}$. Then $\mathcal{A}_{d}=\left(A_{d}, \delta_{d}, d_{\varepsilon}, \tau_{d}\right)$ is an accessible crisp-deterministic fuzzy automaton equivalent to $\mathcal{A}$.

Proof: Let $u, v \in X^{*}$ such that $d_{u}=d_{v}$. Then for every $x \in X$ and $a \in A$ we have that

$$
d_{u x}(a)=\bigwedge_{w \in X^{*}} \tau_{w}(a) \rightarrow d_{u} \circ \delta_{x} \circ \tau_{w}=\bigwedge_{w \in X^{*}} \tau_{w}(a) \rightarrow d_{v} \circ \delta_{x} \circ \tau_{w}=d_{v x}(a),
$$

so $d_{u x}=d_{v x}$, and hence, $\delta_{d}$ is a well-defined mapping. It is evident that $\tau_{d}$ is also a well-defined mapping. Thus, we have that $\mathcal{A}_{d}=\left(A_{d}, \delta_{d}, d_{e}, \tau_{d}\right)$ is an accessible crisp-deterministic fuzzy automaton. According to Theorem 3.1 and definitions of fuzzy languages recognized by a fuzzy automaton and a crisp-deterministic fuzzy automaton we have that

$$
\llbracket \mathcal{A}_{d} \rrbracket(u)=\tau_{d}\left(\delta_{d}^{*}\left(d_{\varepsilon}, u\right)\right)=\tau_{d}\left(d_{u}\right)=d_{u} \circ \tau=\sigma_{u} \circ \tau=\llbracket \mathcal{A} \rrbracket(u),
$$

for every $u \in X^{*}$, and we have proved that $\mathcal{A}_{d}$ is equivalent to $\mathcal{A}$.

The next theorem establishes the minimality of $\mathcal{A}_{d}$.

Theorem 3.3: Let $\mathcal{A}=(A, \delta, \sigma, \tau)$ be a fuzzy automaton over an alphabet $X$ and a complete residuated lattice $\mathcal{L}$. Then, $\mathcal{A}_{d}$ is a minimal crisp-deterministic fuzzy automaton equivalent to $\mathcal{A}$.

Proof: For the sake of simplicity set $\llbracket \mathcal{A} \rrbracket=f$. According to Theorem $4.1[9]$, the derivative automaton $\mathcal{A}_{f}$ of $f$ is a minimal crisp-deterministic fuzzy automaton recognizing $f$, i.e., a minimal crisp-deterministic fuzzy automaton equivalent to $\mathcal{A}$. Therefore, in order to show that the automaton $\mathcal{A}_{d}=\left(A_{d}, \delta_{d}, d_{\varepsilon}, \tau_{d}\right)$ is a minimal crisp-deterministic fuzzy automaton equivalent to $\mathcal{A}$ it is enough to prove that there exists a surjective mapping from $\mathcal{A}_{f}$ to $\mathcal{A}_{d}$.

Let $\phi: A_{f} \rightarrow A_{d}$ be a mapping defined by $\phi\left(f_{u}\right)=d_{u}$. By (18), for any $u, v \in X^{*}$ such that $f_{u}=f_{v}$, we have

$$
\sigma_{u} \circ \tau_{w}=\llbracket \mathcal{A} \rrbracket(u w)=\llbracket \mathcal{A} \rrbracket_{u}(w)=f_{u}(w)=f_{v}(w)=\llbracket \mathcal{A} \rrbracket_{v}(w)=\llbracket \mathcal{A} \rrbracket(v w)=\sigma_{v} \circ \tau_{w},
$$

for every $w \in X^{*}$. Consequently, for any $a \in A$ we have that

$$
d_{u}(a)=\bigwedge_{w \in X^{*}} \tau_{w}(a) \rightarrow \sigma_{u} \circ \tau_{w}=\bigwedge_{w \in X^{*}} \tau_{w}(a) \rightarrow \sigma_{v} \circ \tau_{w}=d_{v}(a),
$$

and hence, $d_{u}=d_{v}$. This means that $\phi$ is a well defined mapping. It is clear that $\phi$ is surjective. Thus, $\mathcal{A}_{d}$ is a minimal crisp-deterministic fuzzy automaton equivalent to $\mathcal{A}$.

Let $\psi$ be a fuzzy relation on the set of states of a fuzzy automaton $\mathcal{A}=(A, \sigma, \delta, \tau)$. According to definitions provided in [12], $A^{\psi}=\left\{\psi^{u} \mid u \in X^{*}\right\}$ is a collection of fuzzy subsets of $A$ given by

$$
\psi^{\varepsilon}=\psi \circ \tau, \quad \psi^{x u}=\psi \circ \delta_{x} \circ \psi^{u}, \text { for all } u \in X^{*}, x \in X,
$$

and $\mathcal{A}^{\psi}=\left(A^{\psi}, \psi^{\varepsilon}, \delta^{\psi}, \tau^{\psi}\right)$ is a crisp-deterministic fuzzy automaton whose transition function and fuzzy set of terminal states are given by

$$
\delta^{\psi}\left(\psi^{u}, x\right)=\psi^{x u}, \quad \tau^{\psi}\left(\psi^{u}\right)=\sigma \circ \psi^{u}, \text { for all } u \in X^{*}, x \in X .
$$

The fuzzy relation $\psi$ is called left invariant if $\sigma \circ \psi \leqslant \sigma$ and $\delta_{x} \circ \psi \leqslant \psi \circ \delta_{x}$, for each $x \in X$, and it is called weakly left invariant if $\sigma_{u} \circ \psi \leqslant \sigma_{u}$, for each $u \in X^{*}$. Clearly, every left invariant fuzzy relation is weakly left invariant. If $\psi$ is reflexive and weakly left invariant, then $\mathcal{A}^{\psi}$ is equivalent to $\mathcal{A}$, and besides, $\sigma_{u} \circ \psi=\sigma_{u}$ 
and $\sigma_{u} \circ \psi^{v}=\sigma_{u} \circ \tau_{v}$, for all $u, v \in X^{*}$. Moreover, if $\psi^{\text {wli }}$ is the greatest weakly left invariant fuzzy relation and $\psi^{\text {li }}$ is the greatest left invariant fuzzy relation on $\mathcal{A}$, then $\mathcal{A}^{\psi^{\text {wli }}} \leqslant \mathcal{A}^{\psi^{\mathrm{li}}} \leqslant \mathcal{A}$ (cf. [12]).

Now, define inductively a family $\left\{\Delta_{u}\right\}_{u \in X^{*}}$ of fuzzy subsets of $A$, as follows:

$$
\Delta_{\varepsilon}(a)=\bigwedge_{w \in X^{*}} \psi^{w}(a) \rightarrow \sigma \circ \psi^{w}
$$

for all $a \in A$, and for all $u \in X^{*}$ and $x \in X$ we set

$$
\Delta_{u x}(a)=\bigwedge_{w \in X^{*}} \psi^{w}(a) \rightarrow \Delta_{u} \circ \psi^{x w}=\bigwedge_{w \in X^{*}} \psi^{w}(a) \rightarrow \Delta_{u} \circ \psi \circ \delta_{x} \circ \psi^{w},
$$

for all $a \in A$. It is easy to check that Theorems 3.1, 3.2 and 3.3 remain valid when $d_{u}$ is replaced by $\Delta_{u}$, which can significantly improve our canonization method since the cardinality of the family $\left\{\psi^{w}\right\}_{w \in X^{*}}$ is smaller than or equal to the cardinality of $\left\{\tau_{w}\right\}_{w \in X^{*}}$, and it may be significantly smaller. Furthermore, even in some cases where the family $\left\{\tau_{w}\right\}_{w \in X^{*}}$ is infinite, the family $\left\{\psi^{w}\right\}_{w \in X^{*}}$ may be finite (see Example 4.13 [12]).

\section{Algorithm and computational examples}

In order to compute the members of the collection of fuzzy sets $\left\{d_{u}\right\}_{u \in X^{*}}$, using formulas (15) and (16), we first need to compute all members of the family $\left\{\tau_{w}\right\}_{w \in X^{*}}$, what is nothing but the construction of the reverse Nerode automaton $\mathcal{A}_{\bar{N}}$ of the fuzzy automaton $\mathcal{A}$ which we want to determinize. Therefore, we can take the construction of $\mathcal{A}_{\bar{N}}$ as the first step in the construction of $\mathcal{A}_{d}$, and then we can proceed with the computation of the collection $\left\{d_{u}\right\}_{u \in X^{*}}$.

The automaton $\mathcal{A}_{\bar{N}}$ can be computed using an algorithm which is derived from Algorithm 4.2 [12] as its particular case. For the sake of completeness we explicitly formulate this algorithm.

Algorithm 4.1 (Construction of the reverse Nerode automaton $\mathcal{A}_{\bar{N}}$ ): The input of this algorithm is a fuzzy finite automaton $\mathcal{A}=(A, \delta, \sigma, \tau)$ with $n$ states, over a finite alphabet $X$ with $m$ letters and a complete residuated lattice $\mathcal{L}$, and the output is the crisp-deterministic automaton $\mathcal{A}_{\bar{N}}=\left(A_{\bar{N}}, \delta_{\bar{N}}, \tau_{\varepsilon}, \tau_{\bar{N}}\right)$.

The procedure is to construct the transition tree of $\mathcal{A}_{\bar{N}}$ directly from $\mathcal{A}$, and during this procedure we use pointers $s(\cdot)$ which points vertices of the tree under construction to the corresponding integers. The transition tree of $\mathcal{A}_{\bar{N}}$ is constructed inductively as follows:

(A1) The root of the tree is $\tau_{\varepsilon}=\tau$, and we put $T_{0}=\left\{\tau_{\varepsilon}\right\}$ and $s\left(\tau_{\varepsilon}\right)=1$, and we compute the value $\tau_{\bar{N}}\left(\tau_{\varepsilon}\right)=\sigma \circ \tau_{\varepsilon}$.

(A2) After the $i$ th step let a tree $T_{i}$ have been constructed, and vertices in $T_{i}$ have been labelled either 'closed' or 'non-closed'. The meaning of these two terms will be made clear in the sequel.

(A3) In the next step we construct a tree $T_{i+1}$ by enriching $T_{i}$ in the following way: for any non-closed leaf $\tau_{u}$ occuring in $T_{i}$, where $u \in X^{*}$, and any $x \in X$ we add a vertex $\tau_{x u}=\delta_{x} \circ \tau_{u}$ and an edge from $\tau_{u}$ to $\tau_{x u}$ labelled by $x$. Simultaneously, we check whether $\tau_{x u}$ is a fuzzy set that has already been constructed. If it is true, if $\tau_{x u}$ is equal to some previously computed $\tau_{v}$, we mark $\tau_{x u}$ as closed and set $s\left(\tau_{x u}\right)=s\left(\tau_{v}\right)$. Otherwise, we compute the value $\tau_{\bar{N}}\left(\tau_{x u}\right)=\sigma \circ \tau_{x u}$ and set $s\left(\tau_{x u}\right)$ to be the next unassigned integer. The procedure terminates when all leaves are marked closed.

(A4) When the transition tree of $\mathcal{A}_{\bar{N}}$ is constructed, we erase all closure marks and glue leaves to interior vertices with the same pointer value. The diagram that results is the transition graph of $\mathcal{A}_{\bar{N}}$.

Suppose that the subsemiring $\mathcal{L}^{*}(\delta, \sigma, \tau)$ of the semiring $\mathcal{L}^{*}=(L, \vee, \otimes, 0,1)$ generated by all membership values taken by $\delta, \sigma$ and $\tau$ is finite and has $k$ elements. Then the reverse Nerode automaton $\mathcal{A}_{\bar{N}}$ has at most $k^{n}$ states, and according to the analysis of computation time of algorithms provided in [12], the computation time of Algorithm 4.1 is $O\left(m n k^{2 n}\right)$. Using the same arguments we conclude that the Nerode automaton of $\mathcal{A}$ also has at most $k^{n}$ states.

Now we provide the following algorithm. 
Algorithm 4.2 (Construction of the automaton $\mathcal{A}_{d}$ ): The input of this algorithm is a fuzzy finite automaton $\mathcal{A}=(A, \delta, \sigma, \tau)$ with $n$ states, over a finite alphabet $X$ with $m$ letters and a complete residuated lattice $\mathcal{L}$, and the output is the crisp-deterministic automaton $\mathcal{A}_{d}=\left(A_{d}, \delta_{d}, d_{\varepsilon}, \tau_{d}\right)$.

The procedure is to construct the transition tree of $\mathcal{A}_{d}$ directly from $\mathcal{A}$, and during this procedure we use pointers $s(\cdot)$ which points vertices of the tree under construction to the corresponding integers. The transition tree of $\mathcal{A}_{d}$ is constructed inductively as follows:

(B1) First we compute all members of the family $\left\{\tau_{w}\right\}_{w \in X^{*}}$, using steps (A1)-(A3) of Algorithm 4.1.

(B2) The root of the tree is $d_{\varepsilon}$, computed using formula (15), and we put $T_{0}=\left\{d_{\varepsilon}\right\}$ and $s\left(d_{\varepsilon}\right)=1$, and we compute the value $\tau_{\bar{N}}\left(d_{\varepsilon}\right)=d_{\varepsilon} \circ \tau$.

(B3) After the $i$ th step let a tree $T_{i}$ have been constructed, and vertices in $T_{i}$ have been labelled either 'closed' or 'non-closed'. The meaning of these two terms will be made clear in the sequel.

(B4) In the next step we construct a tree $T_{i+1}$ by enriching $T_{i}$ in the following way: for any non-closed leaf $d_{u}$ occuring in $T_{i}$, where $u \in X^{*}$, and each $x \in X$ we add a vertex $d_{u x}$ computed using formula (16), and an edge from $d_{u}$ to $d_{u x}$ labelled by $x$. Simultaneously, we check whether $d_{u x}$ is a fuzzy set that has already been constructed. If it is true, if $d_{u x}$ is equal to some previously computed $d_{v}$, we mark $d_{u x}$ as closed and set $s\left(d_{u x}\right)=s\left(d_{v}\right)$. Otherwise, we compute the value $\tau_{d}\left(d_{u}\right)=d_{u} \circ \tau$ and set $s\left(d_{u x}\right)$ to be the next unassigned integer. The procedure terminates when all leaves are marked closed.

(B5) When the transition tree of $\mathcal{A}_{d}$ is constructed, we erase all closure marks and glue leaves to interior vertices with the same pointer value. The diagram that results is the transition graph of $\mathcal{A}_{d}$.

Assume again that the subsemiring $\mathcal{L}^{*}(\delta, \sigma, \tau)$ is finite and has $k$ elements. As we have already said, the collection $\left\{\tau_{w}\right\}_{w \in X^{*}}$ computed in step (B1) has at most $k^{n}$ different members, and the computational time of this step is $O\left(m n k^{2 n}\right)$. Subsequent steps produce the transition tree of a minimal crisp-deterministic fuzzy automaton equivalent to $\mathcal{A}$, which can not be larger than the Nerode automaton $\mathcal{A}_{N}$ of $\mathcal{A}$. As the Nerode automaton $\mathcal{A}_{N}$ has at most $k^{n}$ states, the resulting transition tree for $\mathcal{A}_{d}$ has at most $k^{n}$ internal vertices, and the total number of vertices does not exceed $m k^{n}+1$. In contrast to other determinization algorithms provided in [8], [10], [11], [12], where the most time-demanding part is the check whether the just computed fuzzy set is a copy of some previously computed fuzzy set, here most of the time is spent on computing the fuzzy sets $d_{u}, u \in X^{*}$. Namely, we can write (15) as

$$
d_{\varepsilon}(a)=\bigwedge_{\mu \in A_{\bar{N}}} \mu(a) \rightarrow \sigma \circ \mu,
$$

for all $a \in A$, and (16) as

$$
d_{u x}(a)=\bigwedge_{\mu \in A_{\bar{N}}} \mu(a) \rightarrow d_{u} \circ \mu_{x}
$$

for all $a \in A, u \in X^{*}$ and $x \in X$, where $\mu_{x}$ denotes the $x$-child of $\mu$ in the transition tree of the reverse Nerode automaton $\mathcal{A}_{\bar{N}}$ of $\mathcal{A}$ (if $\mu=\tau_{v}$, for some $v \in X^{*}$, then $\mu_{x}=\tau_{x v}$ ). The computation of any single $d_{u} \circ \mu_{x}$ (and, similarly, of $\left.\sigma \circ \mu\right)$ takes $O\left(n\left(c_{\otimes}+c_{\vee}\right)\right)$ time, and therefore, the computation of the whole collection of values $\left\{d_{u} \circ \mu_{x}\right\}_{\mu \in A_{\bar{N}}}$ takes $O\left(n k^{n}\left(c_{\otimes}+c_{V}\right)\right)$ time, since $\left|A_{\bar{N}}\right| \leqslant k^{n}$. When all values $d_{u} \circ \mu_{x}$ are stored, then the computation of $d_{u x}(a)$ requires time $O\left(k^{n}\left(c_{\rightarrow}+c_{\wedge}\right)\right)$, and the computation of $d_{u x}$ requires time $O\left(n k^{n}\left(c_{\rightarrow}+c_{\wedge}\right)\right)$. Hence, the computation of any single vertice of the transition tree of the automaton $\mathcal{A}_{d}$ requires time $O\left(n k^{n}\left(c_{\otimes}+c_{\vee}+c_{\rightarrow}+c_{\curlywedge}\right)\right)$, and since there are at most $m k^{n}+1$ vertices to be computed, the computation of all verices requires $O\left(m n k^{2 n}\left(c_{\otimes}+c_{\vee}+c_{\rightarrow}+c_{\wedge}\right)\right)$ time. As for all algorithms considered in [12], the check whether the just computed fuzzy set is a copy of some previously computed fuzzy set is performed in time $O\left(m n k^{2 n}\right)$. Therefore, the whole algorithm runs in time $O\left(m n k^{2 n}\left(c_{\otimes}+c_{\vee}+c_{\rightarrow}+c_{\wedge}\right)\right)$, and if the basic operations in $\mathcal{L}$ can be performed in constant time, then this algorithm has the computational time $O\left(m n k^{2 n}\right)$, the same as all other determinization algorithms provided in [8], [11], [12].

The canonization procedure given in Algorithm 4.2 has the same initial stage as the Brzozowski type canonization procedure provided in [10], the computation of the family $\left\{\tau_{w}\right\}_{w \in X^{*}}$, i.e., the states of the 
a)

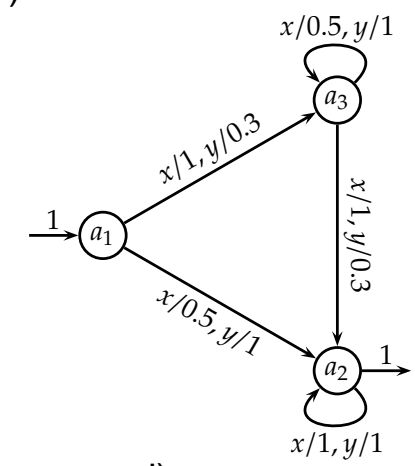

d)

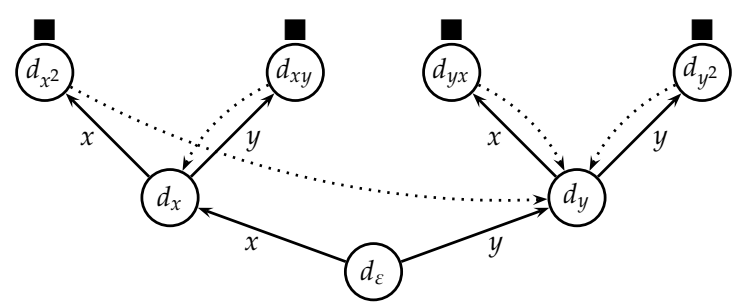

c)
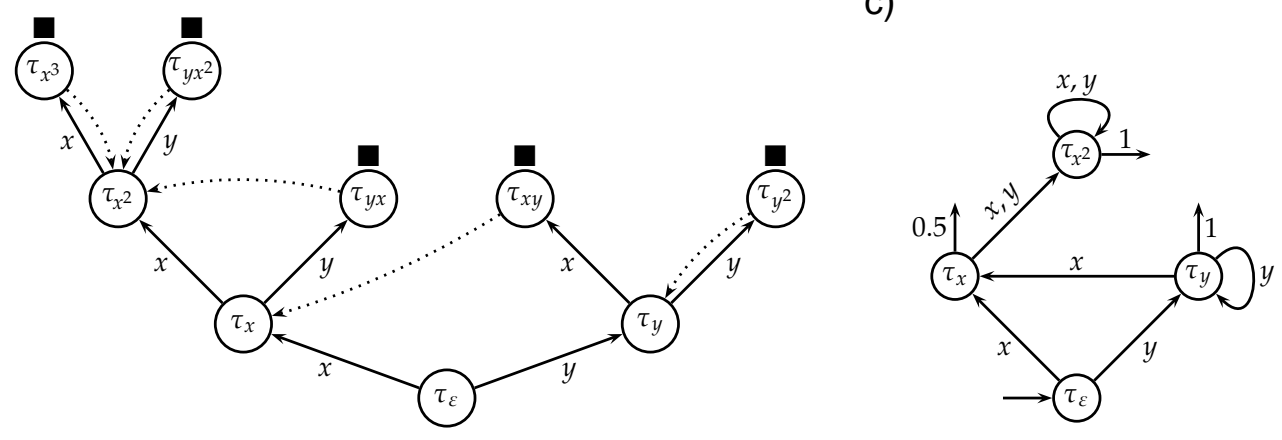

e)

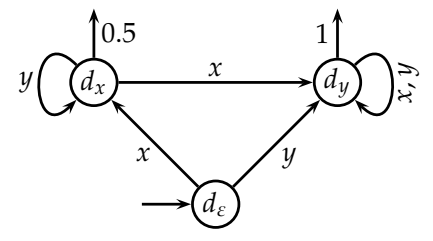

Fig. 1. The transition graph of the fuzzy automaton $\mathcal{A}$ from Example $4.4(\mathrm{a})$ ), the transition tree (b)) and the transition graph (c)) of the reverse Nerode automaton $\mathcal{A}_{\bar{N}}$, and the transition tree (d)) and the transition graph (e)) of the automaton $\mathcal{A}_{d}$

reverse Nerode automaton $\mathcal{A}_{\bar{N}}$. As we have already said, the computation time of this stage is $O\left(m n k^{2 n}\right)$. In the subsequent phases these two procedures differ considerably. While in its latter stages Algorithm 4.2 works with vectors of size $n$, where $n$ is the number of states of $\mathcal{A}$, the Brzozowski type procedure in its latter stages works with vectors of size $r$ and square matrices of size $r \times r$, where $r=\left|\mathcal{A}_{\bar{N}}\right| \leqslant k^{n}$. In its second round the Brzozowski type procedure produces the reverse Nerode automaton of $\mathcal{A}_{\bar{N}}$, which is a minimal crisp-deterministic fuzzy automaton equivalent to $\mathcal{A}$ and it is not greater than the Nerode automaton $\mathcal{A}_{N}$. Thus, this automaton does not have more than $k^{n}$ states, i.e., the resulting transition tree has not more than $k^{n}$ internal vertices, and the total number of vertices is not greater than $m k^{n}+1$. The computation of any single vertex of this transition tree requires time $O\left(k^{2 n}\left(c_{\otimes}+c_{\vee}\right)\right)$, so the time required to compute all vertices is $O\left(m k^{3 n}\left(c_{\otimes}+c_{\vee}\right)\right)$. Since the tree has at most $m k^{n}$ edges, the computation time of their forming is $O\left(m k^{n}\right)$.

When for any newly-constructed fuzzy set we check whether it is a copy of some previously computed fuzzy set, the total number of performed checks is $\frac{1}{2} k^{n}\left(k^{n}+1\right)+(m-1) k^{2 n}$, and the computation time for any single check has $O\left(k^{n}\right)$, so the computation time for all performed checks is $O\left(m k^{3 n}\right)$. Hence, the computation time of the whole algorithm is $O\left(m k^{3 n}\left(c_{\otimes}+c_{\vee}\right)\right)$, or $O\left(m k^{3 n}\right)$, if the operations $\otimes$ and $\vee$ can be performed in constant time. This means that the procedure proposed in Algorithm 4.2 has somewhat better computation time than the Brzozowski type procedure.

Now we give a computational example that demonstrates the application of Algorithm 4.2

Example 4.3: Let $\mathcal{A}=(A, \delta, \sigma, \tau)$ be a fuzzy finite automaton over the two-element alphabet $X=\{x, y\}$ and the Goguen (product) structure, given by the transition graph shown in Fig. 4.3 a). Equivalently, $\mathcal{A}$ is specified by the following matrices and vectors:

$$
\delta_{x}=\left[\begin{array}{ccc}
0 & 0.5 & 1 \\
0 & 1 & 0 \\
0 & 1 & 0.5
\end{array}\right], \quad \delta_{y}=\left[\begin{array}{ccc}
0 & 1 & 0.3 \\
0 & 1 & 0 \\
0 & 0.3 & 1
\end{array}\right], \quad \sigma=\left[\begin{array}{lll}
1 & 0 & 0
\end{array}\right], \quad \tau=\left[\begin{array}{l}
0 \\
1 \\
0
\end{array}\right]
$$

Using step (B1) of Algorithm 4.2 (i.e., Algorithm 4.1) we obtain that the family $\left\{\tau_{w}\right\}_{w \in X^{*}}$ consists of fuzzy 
sets represented by the following vectors:

$$
\tau_{\varepsilon}=\left[\begin{array}{l}
0 \\
1 \\
0
\end{array}\right], \quad \tau_{x}=\left[\begin{array}{c}
0.5 \\
1 \\
1
\end{array}\right], \quad \tau_{y}=\left[\begin{array}{c}
1 \\
1 \\
0.3
\end{array}\right], \quad \tau_{x^{2}}=\left[\begin{array}{l}
1 \\
1 \\
1
\end{array}\right],
$$

since $\tau_{x y}=\tau_{x}, \tau_{y x}=\tau_{x^{2}}=\tau_{x^{3}}=\tau_{y x^{2}}, \tau_{y^{2}}=\tau_{y}$. The transition tree and transition graph of the reverse Nerode automaton $\mathcal{A}_{\bar{N}}$ are given by Fig. $4.3 \mathrm{~b}$ ) and c). In (B2) we first compute products $\sigma \circ \tau_{\varepsilon}=0$, $\sigma \circ \tau_{x}=0.5, \sigma \circ \tau_{y}=1$ and $\sigma \circ \tau_{x^{2}}=1$, and then, for any $a \in A$, we obtain that

$$
\begin{aligned}
d_{\varepsilon}(a) & =\left(\tau_{\varepsilon}(a) \rightarrow \sigma \circ \tau_{\varepsilon}\right) \wedge\left(\tau_{x}(a) \rightarrow \sigma \circ \tau_{x}\right) \wedge\left(\tau_{y}(a) \rightarrow \sigma \circ \tau_{y}\right) \wedge\left(\tau_{x^{2}}(a) \rightarrow \sigma \circ \tau_{x^{2}}\right)= \\
& =\left(\tau_{\varepsilon}(a) \rightarrow 0\right) \wedge\left(\tau_{x}(a) \rightarrow 0.5\right) \wedge\left(\tau_{y}(a) \rightarrow 1\right) \wedge\left(\tau_{x^{2}}(a) \rightarrow 1\right)=\left(\tau_{\varepsilon}(a) \rightarrow 0\right) \wedge\left(\tau_{x}(a) \rightarrow 0.5\right),
\end{aligned}
$$

whence it follows that

$$
d_{\varepsilon}=\left[\begin{array}{c}
1 \\
0 \\
0.5
\end{array}\right], \quad d_{\varepsilon} \circ \tau_{\varepsilon}=0, \quad d_{\varepsilon} \circ \tau_{x}=0.5, \quad d_{\varepsilon} \circ \tau_{y}=1, \quad d_{\varepsilon} \circ \tau_{x^{2}}=1 .
$$

In (B4), for each $a \in A$ we have that

$$
\begin{aligned}
d_{x}(a) & =\left(\tau_{\varepsilon}(a) \rightarrow d_{\varepsilon} \circ \tau_{x}\right) \wedge\left(\tau_{x}(a) \rightarrow d_{\varepsilon} \circ \tau_{x^{2}}\right) \wedge\left(\tau_{y}(a) \rightarrow d_{\varepsilon} \circ \tau_{x}\right) \wedge\left(\tau_{x^{2}}(a) \rightarrow d_{\varepsilon} \circ \tau_{x^{2}}\right)= \\
& =\left(\tau_{\varepsilon}(a) \rightarrow 0.5\right) \wedge\left(\tau_{x}(a) \rightarrow 1\right) \wedge\left(\tau_{y}(a) \rightarrow 0.5\right) \wedge\left(\tau_{x^{2}}(a) \rightarrow 1\right)=\left(\tau_{\varepsilon}(a) \rightarrow 0.5\right) \wedge\left(\tau_{y}(a) \rightarrow 0.5\right), \\
d_{y}(a) & =\left(\tau_{\varepsilon}(a) \rightarrow d_{\varepsilon} \circ \tau_{y}\right) \wedge\left(\tau_{x}(a) \rightarrow d_{\varepsilon} \circ \tau_{x^{2}}\right) \wedge\left(\tau_{y}(a) \rightarrow d_{\varepsilon} \circ \tau_{y}\right) \wedge\left(\tau_{x^{2}}(a) \rightarrow d_{\varepsilon} \circ \tau_{x^{2}}\right)= \\
& =\left(\tau_{\varepsilon}(a) \rightarrow 1\right) \wedge\left(\tau_{x}(a) \rightarrow 1\right) \wedge\left(\tau_{y}(a) \rightarrow 1\right) \wedge\left(\tau_{x^{2}}(a) \rightarrow 1\right)=1,
\end{aligned}
$$

and therefore,

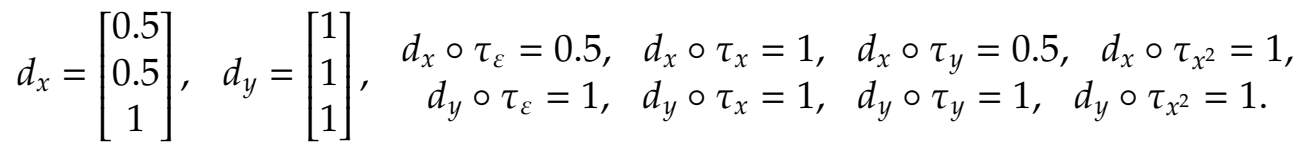

Next, for each $a \in A$ we have

$$
\begin{aligned}
d_{x^{2}}(a) & =\left(\tau_{\varepsilon}(a) \rightarrow d_{x} \circ \tau_{x}\right) \wedge\left(\tau_{x}(a) \rightarrow d_{x} \circ \tau_{x^{2}}\right) \wedge\left(\tau_{y}(a) \rightarrow d_{x} \circ \tau_{x^{2}}\right) \wedge\left(\tau_{x^{2}}(a) \rightarrow d_{x} \circ \tau_{x^{2}}\right)= \\
& =\left(\tau_{\varepsilon}(a) \rightarrow 1\right) \wedge\left(\tau_{x}(a) \rightarrow 1\right) \wedge\left(\tau_{y}(a) \rightarrow 1\right) \wedge\left(\tau_{x^{2}}(a) \rightarrow 1\right)=1=d_{y}(a), \\
d_{x y}(a) & =\left(\tau_{\varepsilon}(a) \rightarrow d_{x} \circ \tau_{y}\right) \wedge\left(\tau_{x}(a) \rightarrow d_{x} \circ \tau_{x^{2}}\right) \wedge\left(\tau_{y}(a) \rightarrow d_{x} \circ \tau_{y}\right) \wedge\left(\tau_{x^{2}}(a) \rightarrow d_{x} \circ \tau_{x^{2}}\right)= \\
& =\left(\tau_{\varepsilon}(a) \rightarrow 0.5\right) \wedge\left(\tau_{x}(a) \rightarrow 1\right) \wedge\left(\tau_{y}(a) \rightarrow 0.5\right) \wedge\left(\tau_{x^{2}}(a) \rightarrow 1\right)= \\
& =\left(\tau_{\varepsilon}(a) \rightarrow 0.5\right) \wedge\left(\tau_{y}(a) \rightarrow 0.5\right)=d_{x}(a), \\
d_{y x}(a) & =\left(\tau_{\varepsilon}(a) \rightarrow d_{y} \circ \tau_{x}\right) \wedge\left(\tau_{x}(a) \rightarrow d_{y} \circ \tau_{x^{2}}\right) \wedge\left(\tau_{y}(a) \rightarrow d_{y} \circ \tau_{x}\right) \wedge\left(\tau_{x^{2}}(a) \rightarrow d_{y} \circ \tau_{x^{2}}\right)= \\
& =\left(\tau_{\varepsilon}(a) \rightarrow 1\right) \wedge\left(\tau_{x}(a) \rightarrow 1\right) \wedge\left(\tau_{y}(a) \rightarrow 1\right) \wedge\left(\tau_{y x}(a) \rightarrow 1\right)=1=d_{y}(a), \\
d_{y^{2}}(a) & =\left(\tau_{\varepsilon}(a) \rightarrow d_{y} \circ \tau_{y}\right) \wedge\left(\tau_{x}(a) \rightarrow d_{y} \circ \tau_{x^{2}}\right) \wedge\left(\tau_{y}(a) \rightarrow d_{y} \circ \tau_{y}\right) \wedge\left(\tau_{x^{2}}(a) \rightarrow d_{y} \circ \tau_{x^{2}}\right)=1=d_{y}(a)
\end{aligned}
$$

so $d_{x^{2}}=d_{y x}=d_{y^{2}}=d_{y}$ and $d_{x y}=d_{x}$, and our algorithm terminates. Its results are the transition tree of the automaton $\mathcal{A}_{d}$, represented by Fig. $4.3 \mathrm{~d}$ ), and the transition graph of $\mathcal{A}_{d}$, represented by Fig. $4.3 \mathrm{e}$ ).

Therefore, the minimal crisp-deterministic fuzzy automaton equivalent to the fuzzy finite automaton $\mathcal{A}$ from this example has 3 states. It is easy to check that algorithms developed in [8], [11], applied to the same fuzzy finite automaton $\mathcal{A}$, yield infinite crisp-deterministic fuzzy automata.

Note that in this case our method gave a finite crisp-deterministic automaton regardless of the fact that the subsemiring $\mathcal{L}^{*}(\delta, \sigma, \tau)$ is infinite.

We also give another example.

Example 4.4: Let $\mathcal{A}=(A, \delta, \sigma, \tau)$ be a Boolean automaton over the two-element alphabet $X=\{x, y\}$ given by the transition graph shown in Fig. 2 a). As in the previous example we construct the automaton $\mathcal{A}_{d}$, whose transition graph is represented by Fig. 2 b). Therefore, the automaton $\mathcal{A}_{d}$, i.e., the minimal crispdeterministic fuzzy automaton equivalent to $\mathcal{A}$ has 4 states. Note that all algorithms developed in [8], [11], [12], applied to the same fuzzy finite automaton $\mathcal{A}$, yield crisp-deterministic fuzzy automata with 7 and 6 states (cf. Example 4.9 in [12]). 
a)

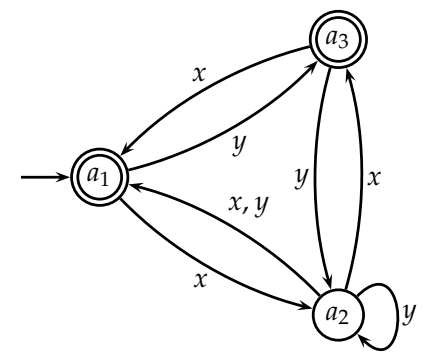

b)

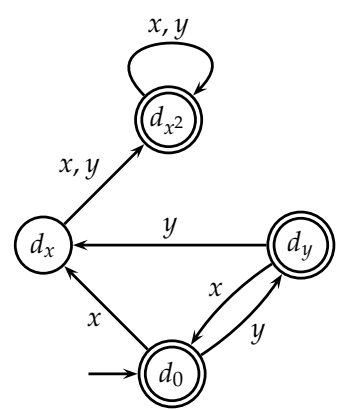

Fig. 2. The transition graph of the fuzzy automaton $\mathcal{A}$ from Example $4.4(\mathrm{a}))$, and the transition graph of the automaton $\left.\mathcal{A}_{d}(\mathrm{~b})\right)$.

\section{References}

[1] R. Bělohlávek, Fuzzy Relational Systems: Foundations and Principles, Kluwer, New York, 2002.

[2] R. Bělohlávek, Determinism and fuzzy automata, Information Sciences 143 (2002) 205-209.

[3] R. Bělohlávek, V. Vychodil, Fuzzy Equational Logic, Studies in Fuzziness and Soft Computing, Springer, Berlin-Heidelberg, 2005.

[4] M. Ćirić, M. Droste, J. Ignjatović, H. Vogler, Determinization of weighted finite automata over strong bimonoids, Information Sciences 180 (2010) 3497-3520.

[5] J. R. González de Mendivil, J. R. Garitagoitia, Fuzzy languages with infinite range accepted by fuzzy automata: Pumping Lemma and determinization procedure, Fuzzy Sets and Systems 249 (2014) 1-26.

[6] J. R. González de Mendivil, J. R. Garitagoitia, Determinization of fuzzy automata via factorization of fuzzy states, Information Sciences 283 (2014) 165-179.

[7] J. Ignjatović, M. Ćirić, Formal power series and regular operations on fuzzy languages, Information Sciences 180 (2010) 1104-1120.

[8] J. Ignjatović, M. Ćirić, S. Bogdanović, Determinization of fuzzy automata with membership values in complete residuated lattices, Information Sciences 178 (2008) 164-180.

[9] J. Ignjatović, M. Ćirić, S. Bogdanović, T. Petković, Myhill-Nerode type theory for fuzzy languages and automata, Fuzzy Sets and Systems 161 (2010) 1288-1324.

[10] Z. Jančić, M. Ćirić, Brzozowski type determinization for fuzzy automata, Fuzzy Sets and Systems 249 (2014) $73-82$.

[11] Z. Jančić, J. Ignjatović, M. Ćirić, An improved algorithm for determinization of weighted and fuzzy automata, Information Sciences 181 (2011) 13581368.

[12] Z. Jančić, I. Micić, J. Ignjatović, M. Ćirić, Further improvements of determinization methods for fuzzy finite automata, Fuzzy Sets and Systems, submitted for publication (http://arxiv.org/pdf/1402.6510v3).

[13] Y. M. Li, W. Pedrycz, Fuzzy finite automata and fuzzy regular expressions with membership values in lattice ordered monoids, Fuzzy Sets and Systems 156 (2005) 68-92.

[14] D. W. Qiu, Automata theory based on completed residuated lattice-valued logic (I), Science in China, Ser. F, 44 (6) (2001) 419-429.

[15] D. W. Qiu, Automata theory based on completed residuated lattice-valued logic (II), Science in China, Ser. F, 45 (6) (2002) 442-452.

[16] D. W. Qiu, Pumping lemma in automata theory based on complete residuated lattice-valued logic: A note, Fuzzy Sets and Systems 157 (2006) 2128-2138.

[17] R. van Glabbeek, B. Ploeger, Five determinization algorithms, in: O.H. Ibarra and B. Ravikumar (eds.), CIAA 2008, Lecture Notes in Computer Science 5148 (2008) 161-170.

[18] R. van Glabbeek, B. Ploeger, Five determinization algorithms, CS-Report 08-14, Eindhoven University of Technology, 2008.

[19] L. H. Wu, D. W. Qiu, Automata theory based on complete residuated lattice-valued logic: Reduction and minimization, Fuzzy Sets and Systems 161 (2010) 1635-1656.

[20] H. Xing, D. W. Qiu, F. C. Liu, Automata theory based on complete residuated lattice-valued logic: Pushdown automata, Fuzzy Sets and Systems 160 (2009) 1125-1140. 